\title{
Singular optics methods for analysis of spatial structure of diffraction field of optical elements
}

\author{
O.P. Budnyk, R.A. Lymarenko \\ International Centre "Institute of Applied Optics", NAS of Ukraine, 10-G Kudryavska str., 04053 Kyiv, Ukraine \\ Phone: +38(044)212 2158, fax: +38(044)2124812,e-mail: myoptics@zeos.net
}

\begin{abstract}
The paper is devoted to developing methods of analytical and experimental investigations of diffraction and interference phenomena used in test systems for optical elements. The theoretical analysis and experimental results illustrate the possibility of describing diffraction phenomena using the objects and methods that were developed in singular optics. It was shown that a system of dislocations in singular component of diffraction field represents its topology. The diffracted field has a system of hidden optical vortices that are smoothly transformed during deformation of an aperture depending on boundary flexion. The proposed experimental proof ground can be useful for the analysis of a wavefront structure. It is also considered the technique for more accurate evaluation of Ronchi test results. The mathematical background of the Ronchi test technique is developed. It describes sufficiently well the wavefront shape, grating plate parameters, image sensor characteristics, parameters of image acquisition and restoration. The fringe pattern distributions and their spatial spectrum are calculated. Both the results of computer simulation of Ronchi fringe pattern and experimental ones obtained using image sensor and the applied image enhancement algorithms are shown.
\end{abstract}

Keywords: singular optics, wavefront shape, Ronchi test.

Paper received 09.12.02; revised manuscript received 12.06.03; accepted for publication 17.06.03.

\section{Introduction}

Recently alongside with the classical interferometer constructed by the principle of division of amplitude of a light wave, works on creation and application of interferometer based on the principle of the wave front replication take place, too. The attention of researchers is involved in these devices, mainly, due to their high stability to vibrations. Besides devices under such circuits do not demand application of reference optical surfaces and allow conducting the control at illumination by white light or with allocation of any wavelength. For the control of optical systems of microscopes the interferometers are advanced on the basis of application low-frequency diffraction gratings. Such scheme was offered in the beginning of the last century to the Italian scientists Vasco Ronchi for the control of astronomical systems. In this paper, we discuss the actual further development of the methods for optical element control.

Special attention is given to singular wave component: edge dislocation wave (D-wave) that was described by the complex Fresnel integral and contains the main numerical information about diffraction process. Thus, the role of introduced edge dislocation wave is beyond the scope of physical interpretation of a rigorous Sommerfield's solu- tion [1-3] of plane wave diffraction on a half-plane. Using the singular component of diffraction field is helpful for investigation of the features of diffraction plane wave on two-dimensional aperture. In this paper, we consider a new method of studying the spatial structure of the diffraction field and describing the determinative singular component of electromagnetic field diffracted on arbitrary two-dimensional apertures. This approach can be used for modelling the diffraction phenomena and studying the creation of optical vortices [4.5] under diffraction on an elliptical aperture and the transformation of hidden dislocation trajectory under smooth deformation of aperture from circular to elliptical one.

\section{Singular approach to diffraction phenomena}

In our previous paper [6,7], was introduced D-wave as an informative part of the diffracted field. The aperture-diffracted field was represented as superposition of two components that exist as real waves: an ordinary wave with amplitude half that of the incident wave and D-wave. The most important feature of this component is zero amplitude on lines corresponding to linear dislocations. The result of incident wave $E_{0}$ diffraction on complementary screens was denoted in the following form: 


\section{O.P. Budnyk, R.A. Lymarenko: Diffraction and interference in wave light ...}

$$
E_{1}=E_{0} / 2+D, \quad E_{2}=E_{0} / 2-D^{\prime}
$$

where $E_{1}$ is the diffraction field for the screen and $E_{2}$ is the field for an obstacle. We note that the introduced D-wave is the same for the aperture and complementary screen in scalar theory: $D=D^{\prime}$. Moreover, the diffraction field for a phase screen with a $p$-phase step coincides with the Dwave for the same screen amplitude. This result revealed inherent relationship of Babinet principle: $E_{1}+E_{2}=E_{0}$. It should be also stated that this is a scalar form of the Babinet theorem, but a vector form has also symmetry feature for electromagnetic field components [8].

The apertured field structure can be analyzed using investigation of singular points in the $\mathrm{D}$-wave. D-wave has a system of linear edge dislocation grouped pairs and the position of optical vortices was determined analytically. The space structure of the dislocation system possesses symmetry properties and the hidden dislocations compose the skeleton of the diffraction field. Studying the spatial evolution of dislocations can help to investigate the topology of the optical field. The structure of the apertured electromagnetic field is easy to imagine as a dislocation wireframe, which are continuously "stretched" so that the aperture is smoothly transform. The smooth deformation of aperture leads to transform the linear dislocation. This effect is similar to transformation of dislocation lines in Gaussian beam after selfinduced Gaussian lens: in case of astigmatic lens we obtain the optical vortice (OV) quadruple. In a general case, the transformation of singular trajectory determines the radius of flexion of aperture boundary. The trajectory is extending from screen while radius is decreased and vice $v e r s a$. Fig. 1 shows the transformation of circle dislocation for round triangle and rectangle apertures.
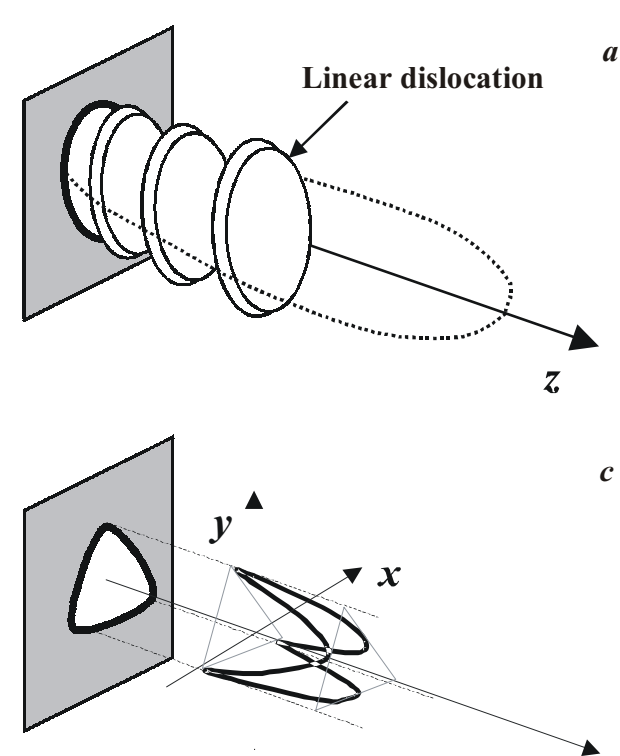

Z.
A priori, it is not clear what happens if an aperture is more complex. For any rounded apertures, we can observe the effect of transversal focusing, i.e. the field oscillations evolve in plane, which is perpendicular to direction of incident wave propagation. The direction of evolution is normal to boundary of an aperture and a radius of curvature of aperture boundary determines the velocity of transversal evolution.

The shape of a wavefront was analyzed and visualized by using the reference plane wave that was slightly tilted with respect to the direction of propagation of the investigated waves. The observed interference fringes were shifted by the half of the period along a dislocation line, it corresponds to the $p$-shift. The dislocations in each pair have an opposite topological "charge" that equals \pm 1 . Therefore, we can see the additional fringes appearing between. The experimental verification of the properties of linear dislocation system in D-wave was performed by subtraction of the plane-wave component from the diffraction field behind the non-transparent screen, in a good agreement with the theoretical predictions. In the frames of Fig. 2, we can observe the quadruple annihilation into dipole and then dislocation disappearing in accord to the spatial trajectory that was schematically shown in Fig. 1d.

The structure of the plane wave diffracted by the rectangular aperture was shown in Fig. 2. The transversal distribution of the amplitude was shown at the distance equals $2 \mathrm{~m}$ for two cases: the square and rectangular with the size ratio $2: 1$. We can see the structure differences between considered cases when illustrating the singular trajectory behaviour that is shown in Fig. 1d. In this context, the longitudinal trajectories shift accords to changing the transverse size of the aperture. It is important that
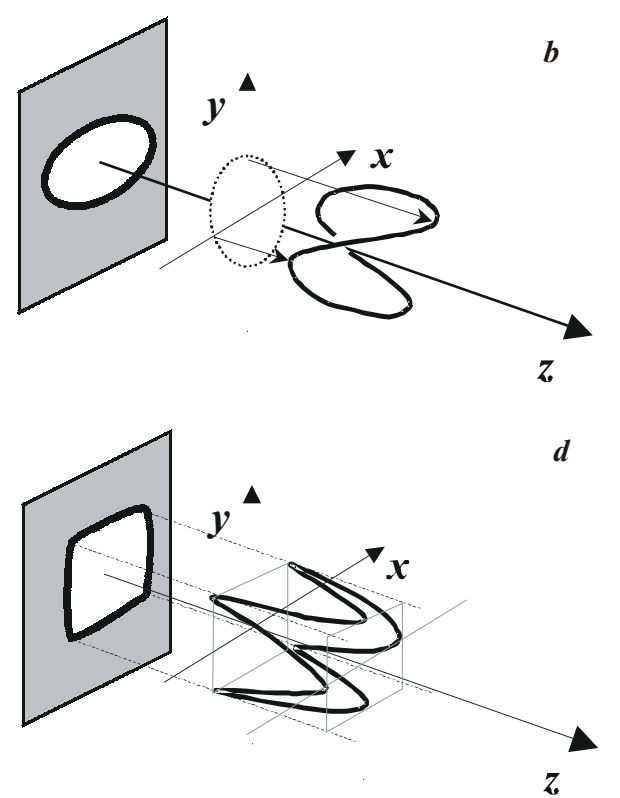

Fig. 1. The spatial structure of the trajectories of linear singularities in D-wave for some kinds of two-dimensional apertures. The transversal dislocation for the circular aperture was transformed while the aperture was smoothly deformed. Schematically shown are the trajectories of dislocations for circular $(a)$, elliptical $(b)$, triangle $(c)$ and rectangular $(d)$ apertures. 


\section{O.P. Budnyk, R.A. Lymarenko: Diffraction and interference in wave light ...}
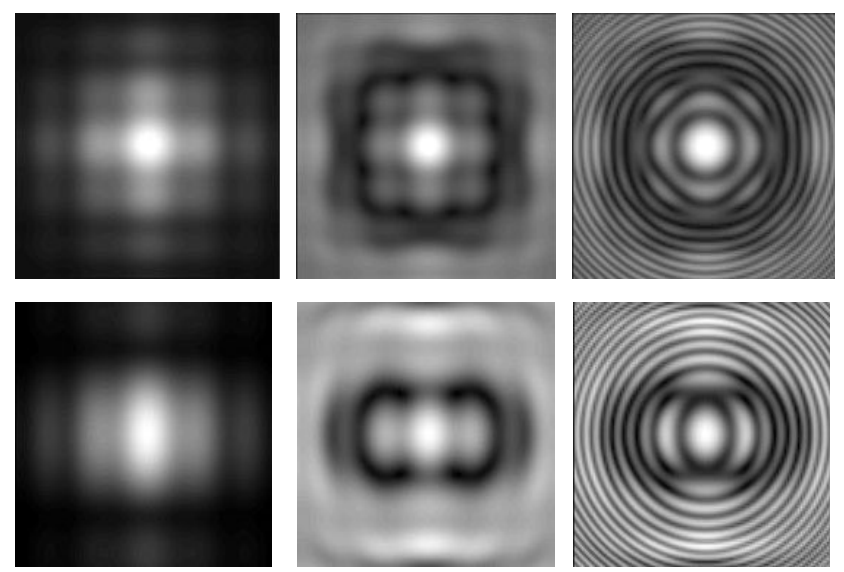

Fig. 2. The amplitude distribution of plane wave diffraction on the rectangular aperture (frame size $4 \times 4 \mathrm{~mm}$, the dotted line shown an aperture boundary). The first column represents the diffraction field, second and third columns represent D-wave and its phase pattern that was visualized using the reference spherical wave.

the behavior of the wave vector around the optical vortices is quite non-paraxial. Therefore, a non-paraxial method of diffraction field modeling is required. In contradiction to another method of solution of the wave equation, the proposed integral representation of diffracted field was based on obtained solution of the problem of plane wave diffraction on rectangular aperture as a product of a slit diffraction function. Using the unit rectangular cell wave representation of two-dimensional aperture, we obtain the diffraction field in the form:

$$
E=E_{0} \times \sum_{n} W_{n} \text {. }
$$

It's easy to see that D-function of the inner unit cell does not take part in field construction. This peculiarity of introduced rectangular unit cell - inter-destruction of field oscillation from joint inside is sufficient decrease of the needed number of elementary cells in (2). This allows to sufficiently decrease the time of computer modelling without losses in accuracy. In the limit case, when the size of unit cell trends to zero, we obtain the expression for the secondary wave W (Unit Cell Wave), which is illuminated by the elementary cell $d s=d \xi d \eta$. Then the expression of diffraction field can be written in the integral form:

$$
\begin{aligned}
& E(x, y, z)=\iint_{A} W d s= \\
& =\iint_{A} \frac{i}{\pi} \frac{\partial U_{\xi}}{\partial \xi} \frac{\partial U_{\eta}}{\partial \eta} \exp \left(i U_{\xi}^{2}+i U_{\eta}^{2}\right) \exp (-i k z) d \xi d \eta,
\end{aligned}
$$

where $U_{\zeta}$ and $U_{\eta}$ are dimensionless curvilinear quasiparabolic coordinates:

$$
U_{\xi}= \pm \sqrt{k} \sqrt{\sqrt{(x-\xi)^{2}+z^{2}}-z}
$$

$$
U_{\eta}= \pm \sqrt{k} \sqrt{\sqrt{(y-\eta)^{2}+z^{2}}-z}
$$

and sign "+" is used for $x-\xi>0(y-\eta>0)$ and vice versa. In paraxial approximations Exp. (3) transforms into the well-known Rayleigh-Sommerfeld solution for two-dimensional problems with Dirichlet conditions and Kirchhoffs approximation and also clearly illustrates the Huygens-Fresnel principle. Due to specific form of the convolution kernel, the aperture integral (3) can be reduced to the multiple one and moreover to be simplified using the symmetry of the considered problem. This speciality allows obtaining the solution in an analytical form in some cases.

\section{Technique for accuracy evaluation of the Ronchi test}

\subsection{Geometrical and interferential aspects of the Ronchi test}

In this centennial of Vasco Ronchi's birth it seems appropriate to devote one of these columns to the well-known method of testing optical systems that he developed in 1920 's $[1,2]$. The essential features of the Ronchi test may be described by the reference to Fig. 3. A lens (or more generally, an optical system consisting of a number of lenses and mirrors) is placed in the position of the "object under test". The lens is then illuminated with a beam of light, which, for the purposes of the present article, will be assumed to be coherent and quasi-monochromatic.

The lens brings the incident beam to a focus, in the vicinity of which a diffraction grating is placed perpendicularly to the optical axis. (The optical axis will be denoted as Z-axis throughout this article.) The grating, also referred to as a Ronchi ruling, may be as simple as a low-frequency wire-grid, or as sophisticated as a modern short-pitched, phase/amplitude grating. The position of the grating should be adjustable in the vicinity of focus, so that it may be shifted back and forth along the optical axis. The grating breaks up the incident beam into multiple diffracted orders, which will subsequently propagate along Z (Fig. 3). (The pupil relay may simply be the lens of the eye, which projects the exit pupil of the object under test onto the retina of the observer. Alternatively, it may be a conventional lens that creates a real image of the exit pupil on a screen or CCD camera.) The diffracted orders from the grating will be collected by the relay lens and, within their overlapping areas, will create interference fringes characteristic of the aberrations of the optical system under consideration. By analyzing these fringes, one can determine the type and, with some effort, the magnitude of the aberrations present at the exit pupil of the system. The above description of the Ronchi test relied on its modern interpretation based on our current understanding of physical optics and the theory of diffraction gratings [9-13]. 


\section{O.P. Budnyk，R.A. Lymarenko: Diffraction and interference in wave light ...}

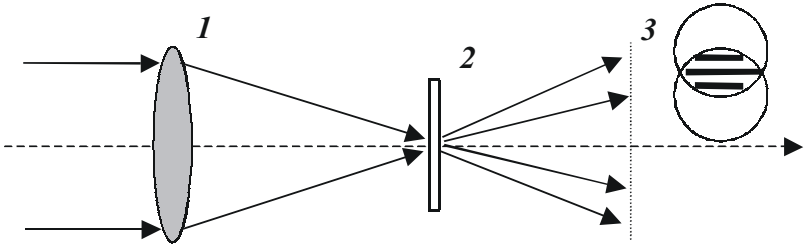

Fig. 3. A beam of coherent, quasi-monochromatic light is brought to focus by an optical system that is undergoing tests to determine its aberrations. A diffraction grating 2, placed perpendicular to the optical axis in the vicinity of focus, breaks up the incident beam into several diffraction orders. The diffracted orders propagate, independently of each other, and are collected by a pupil relay lens, which forms an image of the exit pupil of the object under test at the observation plane 3 .

Several modifications and extensions of the Ronchi test have appeared over the years, and have helped to solve specific problems in testing of optical systems. As an example, we mention the "double-frequency grating lateral shear interferometer" invented by James Wyant in the early 1970's. The grating in this device has two slightly different frequencies, which give rise to two $+1^{\text {st }}$ order beams as well as two $-1^{\text {st }}$ order beams; the beams in each pair are slightly shifted relatively to each other. Moreover, the (average) pitch of the grating is such that there is no overlapping between the 0 th,$+1^{\text {st }}$, and $-1^{\text {st }}$ orders. Consequently, interference occurs between the two $+1^{\text {st }}$ order beams (and, likewise, between the two $-1^{\text {st }}$ order beams). One can thus obtain an arbitrarily small lateral shear of the wavefront under test, and use the results to achieve accurate quantitative measurements.

\subsection{The experimental results and computer modeling of Ronchi fringe pattern}

For computer simulation of aberrations field we use Zernike polynomial in the following form:

$$
\begin{aligned}
& Z_{n}^{l}=R_{n}^{l}(\rho) e^{i l \theta}, \\
& R_{n}^{n-2 m}=\sum_{s=0}^{\min \left(m,\left[\frac{n}{2}\right]\right)}(-1)^{s} \frac{(n-s) !}{s !(m-s) !(n-m-s) !} \cdot \rho^{n-2 s},
\end{aligned}
$$

where $n$ is a degree of polynomial, $l$ is an angular parameter, $\theta$ is a polar angle, $\rho$ is a normalized radial distance, $m=(n-l) / 2$ is a positive number. The arbitrary function (wavefront) $W(\rho, \theta)$ with $\mathrm{K}$ degree can be represented as the linear combination of the radial Zernike polynomial:

$W(\rho, \theta)=\sum_{n=0}^{k} \sum_{l=-n}^{n} C_{n l} R_{n}^{|l|} e^{i l \theta}$, or

$$
\begin{aligned}
& W(\rho, \theta)=\sum_{n=0}^{k} \sum_{m=0}^{n} A_{n m} U_{n m}= \\
& =\sum_{n=0}^{k} \sum_{m=0}^{n} A_{n m} R_{n}^{n-2 m}\left\{\begin{array}{c}
\sin \\
\cos
\end{array}\right\}(n-2 m) \theta,
\end{aligned}
$$

where sin corresponds to $n-2 m>0$, and $\cos$ under $n-2 m \leq 0$, $C_{n, 1}=A_{n,(n+1) / 2}$ are coefficients in series development for $W(\rho, \theta)$.

Usually the wavefront function was written as monomial, i.e. series of $\mathrm{x}$ and $\mathrm{y}$ degrees:

$W(x, y)=\sum_{i=0}^{k} \sum_{j=0}^{i} B_{i j} x^{j} y^{i-j}$

Such function was appeared when the data processing is provided by using the least-squares method. It is possible to transform every Zernike polynomial into the form that corresponds to Exp. (8):

$$
\begin{aligned}
& \left\{\begin{array}{l}
\cos \\
\sin
\end{array}\right\}(n-2 m) \theta=\rho^{-(n-2 m)} \times \\
& \times \sum_{j=0}^{q}(-1)^{j}\left(\begin{array}{c}
n-2 m \\
2 i+p
\end{array}\right) x^{2 j+p} y^{n-2 m-2 j-p},
\end{aligned}
$$

where $p$ and $q$ are parameters that depend on $n$ and $m$.

It is possible to draw the following features about Ronchi test:

sensitivity of the Ronchi method essentially depends on the circuit of the control, parameters of a controllable detail, quality of the photoreception device;

the qualitative control based on Ronchi method is rather rough and may be used only at initial stages of optics processing;

the quantitative control test using the Ronchi method may be carried out and satisfy to Rayleigh's criterion only at presence of the high-quality photoreception device, allowing to provide transfer of a curvature of fringes pattern and mounts providing exact installation of a diffraction grate.

The problem of the computerized Ronchigram analysis to be solved is difficult. Because of the traditional methods are not accurate enough to give reliable results. Instead of more complex analysis of Ronchigrams, we propose to analyze the spatial spectrum of obtained interferogam, i.e. fringes pattern distribution. The computer simulation showed that the maximum of amplitude is localized in the spatial frequency area for different types of aberrations. This method is more convenient for providing the computer data processing. The carried out researches have shown that the given method is perspective and, with all its limitations, the procedure is still applicable. Experimental data and analytical results are found to be in a good agreement. 
O.P. Budnyk, R.A. Lymarenko: Diffraction and interference in wave light ...

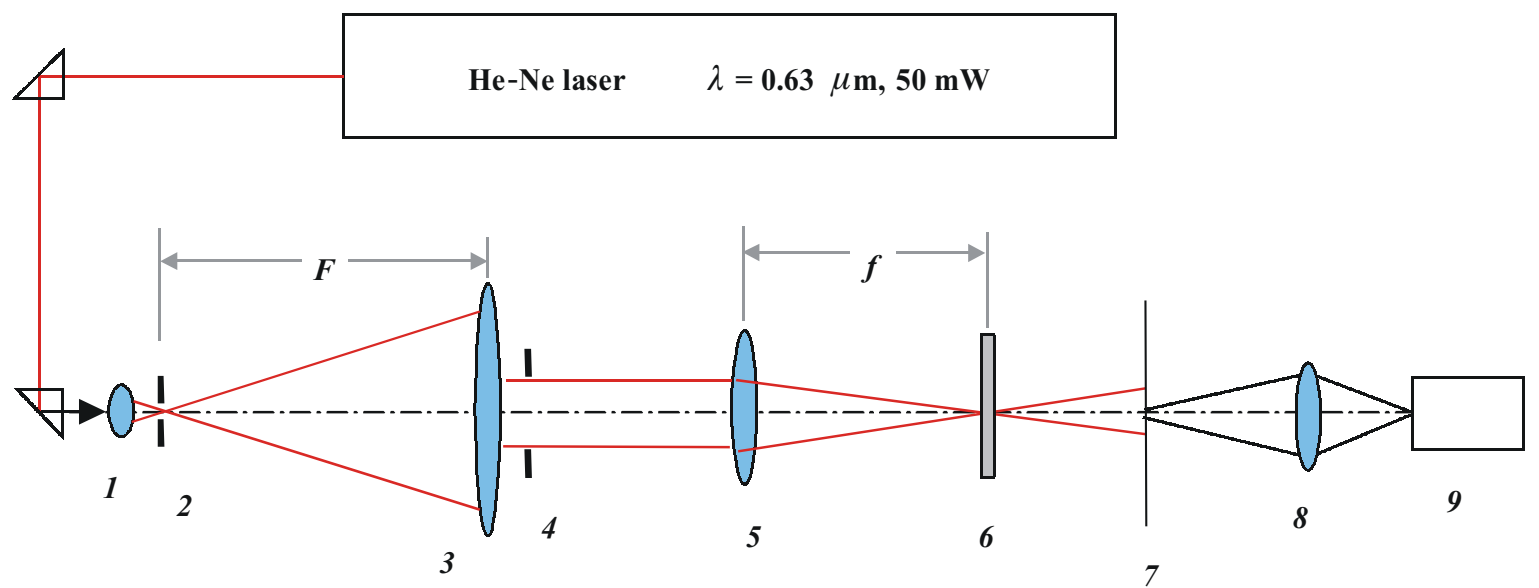

Fig. 4. Experimental setup: $I-$ microobjective, 2 - pin-hole $20 \mathrm{~mm}, 3$ - objective $D=200 \mathrm{~mm}, F=100 \mathrm{~cm}, 4-\operatorname{diaphragm~} D=20$ $\mathrm{mm}, 5$ - test-objective $f=21 \mathrm{~cm}, 6$ - diffraction grate $d=15 \mu \mathrm{m}, 7$ - observation plane, 8 - objective, 9 - CCD camera.
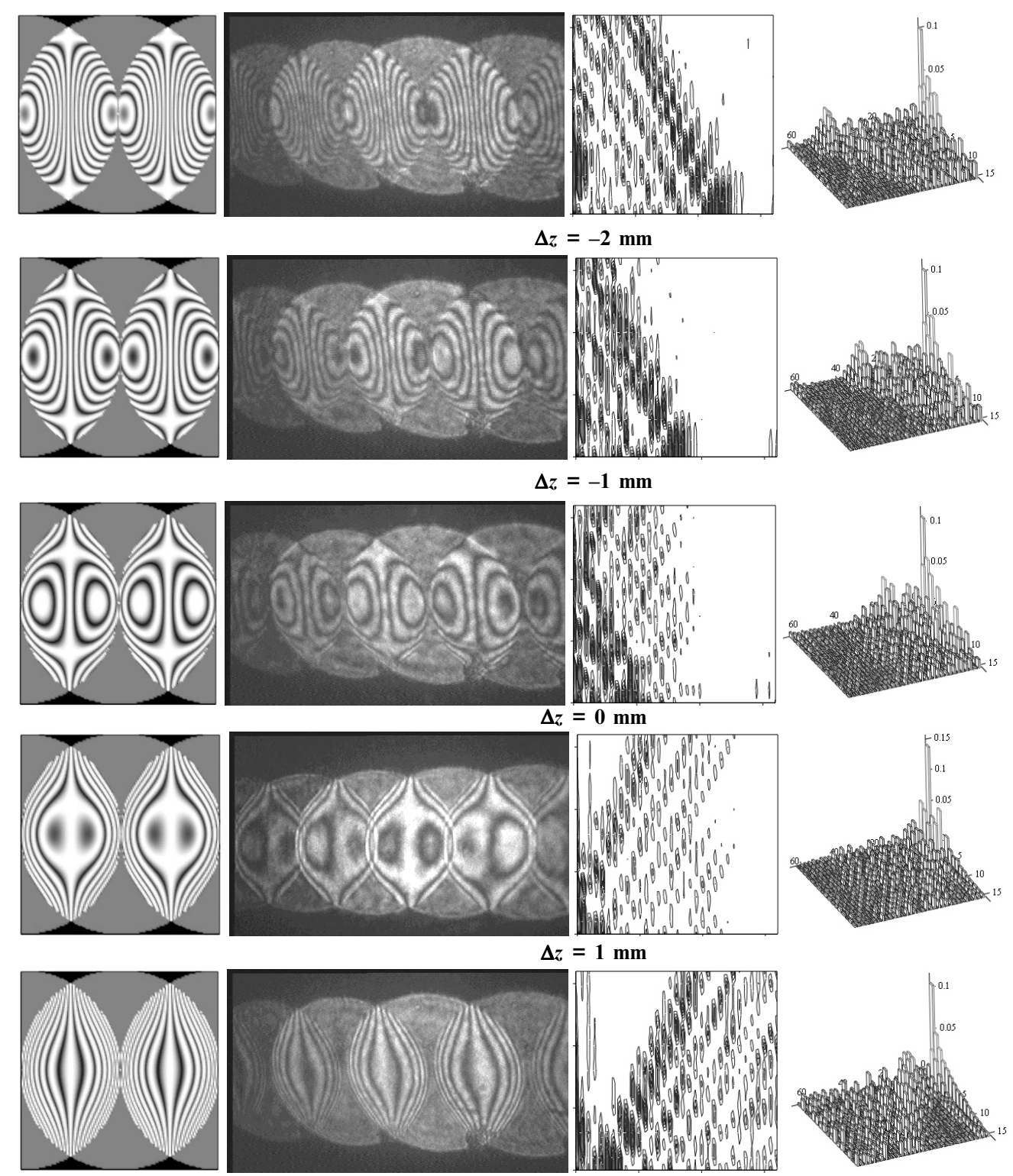

$\Delta z=2 \mathrm{~mm}$

Fig. 5. Sliding the grating along the optical axis (aberration $n=4 m=2$ ) (modeling, experimental Ronchigram and its spatial spectrum). 


\section{O.P. Budnyk, R.A. Lymarenko: Diffraction and interference in wave light ...}

\section{Conclusions}

The paper represents the technique for wavefront structure analysis for testing of optical elements. The theoretical analysis and experimental results illustrate the possibility of describing diffraction phenomena using the objects and methods that were developed in singular optics. It was shown that a system of hidden dislocation lines composes the skeleton and represents the diffraction field topology. The singular trajectory evolution for rounded apertures was discussed. It was shown that the trajectories evolve in direction that coincides to direction of incident wave propagation and an aperture deformation determines the longitudinal scaling. Due to this, the investigation of spatial evolution of aperture-diffracted field is reduced to studying the transformation of system of closed pairs of linear dislocation in singular component. This approach to the problem can be useful in the explanation of other diffraction effects, in practical applications, and even in revealing new phenomena in this traditional area of optics. The totality of experimental results substantiated the theoretical conclusions. The paper also represents the technique for accuracy evaluation of Ronchi test of optical elements. Proposed method of Ronchigramm analysis in spatial frequency area is not optimal due to partition of spatial spectrum component of different Zernike polynoms. It was proposed the wavelet transform using for optimal filtration and recognition instead of the Fourier transform that is more convenient for practical realization of measuring system based on analysis of orientation and spatial position of Ronchigram elements.

\section{References}

1. M. Born, E.Wolf, Principles of Optics, 6-th ed. Pergamon Press, Oxford, 1991.

2. A. Sommerfeld, Mathemathishe theorie der diffraction // Math. Annalen, 47, pp. 317-374 (1896).

3. R. Vaganov, B. Katzelenbaum. Diffraction theory, M. Nauka, 1982.

4. J.F. Nye and M.V. Berry, Dislocations in wave trains // Proc. R. Soc. Lond. A 336, pp. 165-190, 1974.

5. N.B. Baranova, B.Ya. Zel'dovich, Dislocations of Wave-Front Surfaces and Amplitude Zeros // ZhETF, 80, pp. 1789-1797 (1981) [Sov. Phys. JETP 53, pp. 925-931 (1981)].

6. A.I. Khizhnyak, S.P. Anokhov, R.A. Lymarenko, M.S. Soskin, M.V. Vasnetsov, The structure of edge-dislocation wave originated in plane-wave diffraction by a half-plane // $\mathrm{J}$. Opt. Soc. Am. A. 17, No. 12, pp. 2199-2207 (2000).

7. R.A. Lymarenko, M.S. Soskin, A.I. Khizhnyak, S.P. Anokhov, The properties and space evolution of hidden singularities in apertured electromagnetic field // Proc. SPIE, 4403, pp.145152 (2000).

8. J.D. Jackson, Classical electrodynamics, John Wiley \& sons, Inc., New York - London, 1962.

9. D. Gabor, Microscopy by reconstructed wavefronts. II // Proc. Phys. Soc., B64, pp. 449-469 (1951).

10. D. Malacara, A.Cornejo, A. Morales, Computation of Zernike Polynomials in Optical testing // Bol. Inst. Tonantzintla, 1(2), P. 21 (1976)

11. F. Zernike, Buegungstheorie des Schneidenver-Eanrens und Seiner Verbesserten Form, der Phasenkontrastmethode, Physica, 1, P. 698 (1934).

12. H.H. Hopkins, Wave Theory of Abberations, Claredon Press, Oxford, 1950

13. Masud Mansuripur, The Ronchi Test // Optics \& Photonics News, pp. 42-46 (1997). 